pictures, to everybody concerned (from politicians and senior Civil servants to workers on the shopfloor); that we should take pains to let people know what our functions are; that we should constantly strive to acquire more appreciation of the problems of the managers we advise ; and that, above all, we should investigate thoroughly the causes of managerial and shop-floor resistance to techniques intended to increase production. He suggested that the British Association should later stage a conference with a broader sweep, at which a series of short progress reports might be read on 'fitting the man to the job' and 'fitting the job to the man'. Under the first heading might come work on the improvement of methods of vocational guidance, personnel selection, and occupational training. Under the second might come the improvement of methods of work, design and layout of working equipment, and working conditions (both physical and psychological).

\section{SCIENTIFIC RADIO}

A SPECIAL feature of the programme of the Radio Section of the Institution of Electrical Engineers this session was the holding of a Convention on Scientific Radio during April 7 and 8. This Convention, which was organised by the Institution in co-operation with the British National Committee for Scientific Radio, afforded an opportunity for a preliminary survey of the British contribution to be presented to the forthcoming meeting of the Union Radio Scientifique Internationale (U.R.S.I.), which has already been announced in Nature of May 1, p. 695. The proceedings of the Convention were divided into four sessions corresponding in scope to the four commissions of the Union Radio Scientifique Internationale; and in each session four or five short papers were presented surveying progress in the past few years in various parts of the field of fundamental radio science. Ample opportunity was provided for speakers who wished to discuss the individual contributions, and abstracts of the papers and of the discussions will in due course be published in the Journal of the Institution.

\section{Standards and Measurements}

The first session, on "Standards and Measurements", was held under the chairmanship of Mr. P. Good, president of the Institution of Electrical Engineers; and the four papers presented on this occasion emanated from the National Physical Laboratory (Department of Scientific and Industrial Research). The chairman explained that this was perhaps to be expected, since it is one of the duties of the Laboratory to set up and maintain standards of reference for the electrical and radio measurements required by all users of the corresponding techniques. As was made quite clear by the individual speakers who followed, however, it was not intended to suggest that all progress made in recent years had been conducted within the Department of Scientific and Industrial Research; for it is well known that many substantial contributions during the war years were made by certain Government establishments and by industry.

Dr. L. Hartshorn, in a paper on "Radio Standards" presented jointly with Dr. L. Essen, first referred to the fact that, of the three standards recognized throughout the whole field of physical science, namely, length, mass and time, the last, if not already a radio standard, is very little short of it. For if once it is decided that the vibrations of the exist. ing quartz-frequency standards are more regular than the rotation of the earth, then, for the period in question, the rotating earth is no longer to be regarded as the standard of either frequency or physical time. The paper pointed out the dependence of radio measurements on the existing electrical standards, and emphasized the need for distinguishing between definitive standards, derived standards, transfer standards and working standards. Reference was made to the changes which took place on January 1 last, when the ohm was defined by reference to the standards of length and time, with consequential changes in the numerical values of reactance and resistance of 0.05 per cent. Since inductance and capacitance standards can now be measured at radio frequencies with an accuracy of about 0.01 per cent, these changes are of decided significance in precision radio measurements. Frequency can be measured to a much higher order of accuracy, and an illustration was shown of relative measurements on three typical quartz standards, differing widely in design. These were found to agree with one another to within 2 or 3 parts in $10^{8}$ over a period of several months.

In the following paper, Dr. T. I. Jones reviewed the techniques used in the measurement of current, voltage, impedance and attenuation at frequencies below about $300 \mathrm{Mc} / \mathrm{s}$. Signal generators and receivers are calibrated in terms of voltage which, at a level of about 0.1 volt, can conveniently be measured by means of valve or crystal-detector voltmeters, and by a combination of a vacuum thermojunction and resistors in series or parallel. Suitable voltage-dividers are available to extend the range upwards and downwards. The methods available for measuring impedance fall into two classes : those which use resonance circuits and which are used for measuring components, cables and the properties of dielectrics; and bridge networks, which are available up to frequencies of the order of $50 \mathrm{Mc}$. $/ \mathrm{s}$. but which deteriorate in performance for frequencies above $10 \mathrm{Mc} . / \mathrm{s}$.

Mr. F. M. Colebrook described the changes in technique which have taken place in recent years in conducting radio measurements at frequencies above about $300 \mathrm{Mc} . / \mathrm{s}$. ; and some discussion ensued on the possibility of reaching agreement among workers interested on the value of this boundary frequency, above which current and voltage measurement should give place to the determination of power. The general position with regard to measuring technique at the very high frequencies now in common use remains substantially as has been described in various papers published in the Journal of the Institution of Electrical Engineers during the past two years.

The subject of radio field-strength measurement is of direct interest in connexion with all applications of radio-wave technique, and Dr. R. L. Smith-Rose presented a survey of the main lines of development of this subject over the past ten years or so. At the present time suitable field-strength measuring equip. ments have been developed on an experimental basis for all radio frequencies between about $0 \cdot 1$ and $600 \mathrm{Mc}$./s. ; but there is a need for further development of these and for an inter-comparison of the different types available on both a national and an international basis. 


\section{Propagation}

Sir Edward Appleton, secretary of the Department of Scientific and Industrial Research, presided at the next session, at which five papers were presented dealing with some topical aspects of the subject of radio wave propagation. Dr. A. C. B. Lovell gave an interesting account of his recent investigations on meteors, conducted by the aid of radar techniques at wave-lengths of a few metres and less. When a meteor enters the earth's atmosphere, it burns away at a height of about $100 \mathrm{~km}$. and creates a dense concentration of electrons which scatter energy from incident radio waves. On a wave-length of about $4 \mathrm{~m}$., the number of echoes which have been detected by available radio equipment is very closely related to visible meteoric phenomena. As the wave-length is increased, however, the rate at which echoes are detected undergoes a striking change, and at wave. lengths greater than $8 \mathrm{~m}$. several hundred echoes per hour are received, a number which bears no close relation to the visible observations. The sporadic ionization in the $E$-layer of the ionosphere is now considered to be mainly caused by the effect of meteors, particularly in temperate latitudes.

In 1934 it was discovered that radio waves traversing the ionosphere could interact with each other in such a way that the modulation of one was transferred to the other. This effect was explained at the time by supposing that the absorption of energy from one of the waves increases the temperature of the electrons in the ionosphere, with a consequential increase in their collision frequency with neutral molecules. As a result, the absorption of the other wave is increased; and since this absorption varies with the modulation of the first wave, this modulation will be transferred to the second wave. Experimental work on this phenomenon has recently been resumed at the Cavendish Laboratory at Cambridge; and Mr. J. A. Ratcliffe described the manner in which he and his colleagues have been observing the phase of the transferred modulation, and comparing it with the phase of the modulation received direct, as a ground wave, from the interacting station. From the results of such measurements, it is possible to determine the collision frequency of the electrons at a known height; and the experiments have given values around $5 \times 10^{5} /$ sec. for a height of about $85 \mathrm{~km}$.

The third paper at this session was presented by Dr. W. J. G. Beynon, who gave an account of recent investigations on the reflexion of radio waves from the ionosphere at oblique incidence. He pointed out that it has long become established practice to calculate the maximum usable frequency for any desired transmission path from normal-incidence ionospheric data with an accuracy which is satisfactory for most practical purposes. Experiments on the absorption of radio waves carried out in Great Britain have shown that there is a marked disagreement between the apparent absorption at oblique incidence and that predicted on theoretical grounds from the values measured at normal incidence. In a similar manner, estimates of oblique-incidence ionospheric absorption obtained from observations of signal strength over trans-Atlantic radio circuits disagree with the values expected from long-term normal-incidence measurements. This subject of the absorption of radio waves in the different regions of the ionosphere is being actively pursued at the present time under the auspices of the Radio Research
Board of the Department of Scientific and Industrial Research.

The need for increased knowledge on the propagation of very long radio waves has recently come into prominence due to the development during the War of some novel and very accurate methods of defining the position of a radio receiver, by measurements made on the arriving waves. Work on this subject was in progress at the Cavendish Laboratory before the War; and Mr. R. N. Bracewell gave an account of the experiments, which have now been resumed, on frequencies of 16 and between 30 and $40 \mathrm{kc} . / \mathrm{s}$., corresponding to wave-lengths of about 19,000 and $9,000 \mathrm{~m}$. respectively. The height of reflexion of these very long waves is about $80 \mathrm{~km}$., with regular diurnal variations, which are sym. metrical about noon, of some $18 \mathrm{~km}$. in summer and $12 \mathrm{~km}$. in winter. Such measurements are made by determining the phase path of the sky wave received at distances of a few hundred kilometres, using the ground wave, which varies little with respect to the transmitter, as the source of reference.

Dr. H. G. Booker presented a new way of visual. izing the Sommerfeld theory of propagation over a flat and imperfectly reflecting earth. This theory arose because the-ray theory involves the assumption that the Fresnel reflexion coefficient of the earth does not vary rapidly with angle of incidence, and this assumption is not satisfied for vertically polarized medium waves as used for broadcasting, arriving at the earth's surface at glancing incidence. The main object of the new presentation is to facilitate the solution of problems involving propagation near the surface of the earth partly over land and partly over sea, a condition which applies frequently in practice to radio transmission for communication and other purposes.

\section{Radio Noise}

At the third session, on "Radio Noise", the chair was taken by Sir Robert Watson-Watt, who is chairman of the British National Committee for Scientific Radio. In a paper entitled "A Survey of Recent Progress in the Study of Fluctuation Noise", Dr. D. K. C. MacDonald referred to the report made by the Union Radio Scientifique Internationale on this subject in 1946, and to the subsequent publication of the associated work primarily directed to war requirements, which was concerned broadly with noise at frequencies of 60,600 and from 3,000 to $10,000 \mathrm{Mc} . \mathrm{s}$. The main problems have been in the development of methods and instruments for noise measurements, of valves, circuits and crystal detectors to improve the ratio of signal to noise. It is well established that certain elements show increased noise at relatively low frequencies; and a study of this phenomenon, which includes 'flicker effect' in valves and 'current noise' in semi-conductors, is of considerable significance, for example, in the design of amplifiers for the study of physiological effects.

During the past few years, there has been a marked resumption of interest in 'atmospherics', a subject which had hitherto been rather neglected by radio physicists for some ten years. Two papers presented to the Convention served to direct attention to the investigations now in progress. In the first of these, Dr. T. W. Wormell and Mr. E. T. Pierce described some recent studies made at the Solar Physics Observatory, Cambridge, on the structure of the disturbances in the vertical electric field at the earth's surface resulting from lightning discharges. 
In one type of investigation cathode-ray tube equipment and photographic recording technique are used to investigate the variation with time of the electric field produced by discharges not more than $50 \mathrm{~km}$. distant. Concurrently, an investigation is being made of the detailed wave-form of the disturbance due to radiation from discharges at distances of many hundreds of kilometres. The results of such observations were discussed with the aid of photographic records and diagrams.

In the second paper, Dr. H. A. Thomas, of the National Physical Laboratory, described some of the results obtained from an analysis of observations of the strength of atmospherics as measured at some fourteen stations in various parts of the world. A large number of slides were shown to illustrate the general trend of these observations, which were made on five frequencies between 2.5 and $20 \mathrm{Mc}$./s., in which band the propagation of radio waves through the ionosphere is likely to play an important part in determining the general strength of atmospheric noise at any place. One of the important conclusions from this study, however, is that the characteristics of the atmospheric noise observed at any one location do not appear to be applicable over any large area; and this would tend to indicate that the sources of the noise being measured are local to the observer. Large differences between the ratio of day and night values are observed at different places; seasonal effects are marked, and also differ appreciably with location. Far more data on this subject are required, however, before any very definite conclusions can be drawn on such a complex subject.

The remaining two papers in this session dealt with recent investigations of the characteristics of galactic and solar radiation. Mr. J. S. Hey described how the emission of high-intensity radio waves from sunspot regions was first established by observations made with Army radar receivers in February 1942, during the transit of a large and active spot across the sun's disk. In the intervening years many workers have made observations of this phenomenon, particularly during the recent maximum of the sunspot cycle. The intensity of the radiations observed is particularly strong for a wave-length around 5 metres, and falls off very considerably as the wave-length is reduced below $1 \mathrm{~m}$. Corresponding reception of this type of radio noise, as first noted by Jansky in 1932, has also been studied by recent workers; and in the discussion of this paper, Dr. J. L. Pawsey described some interesting observations, made in Australia, on galactic noise, from which it appears that some of the stars, or combinations thereof, radiate much more strongly than sunspots. This was followed up by Mr. M. Ryle's paper, describing experiments made on radiation emitted by the sun on wave-lengths of 1.7 and $3.8 \mathrm{~m}$. The observations have been continued over a period of fifteen months, and it has been found that in the absence of sunspots the sun radiates with an intensity corresponding to a surface temperature of $10^{\circ}{ }^{\circ} \mathrm{K}$. The radiation from sunspots is characterized by sudden increases of intensity which correspond to much higher temperatures.

\section{Radio Physics}

At the fourth session of the Convention, on "Radio Physics", the chair was taken by Prof. G. I. Finch, president of the Physical Society. The papers presented at this session demonstrated the manner in which some physicists are using the very high. frequency radio techniques, corresponding to wave- lengths of a few centimetres and less, to study various properties of solids, liquids and gases. Dr. B. Bleaney described the use of centimetre waves for studying absorption lines in the spectrum of gases due to heavy polar molecules. By making the radio frequency measurements to a high order of accuracy, the broadening of the lines due to collisions has been investigated at pressures up to six atmospheres. At low pressures $(0 \cdot 1-10 \mathrm{~cm}$. mercury), the widths of the lines vary directly with the pressure; while at pressures above one atmosphere, the line breadth ceases to rise as rapidly as the pressure. Among the most interesting spectra studied in this manner are those of ammonia and water-vapour, the latter being of particular importance as a constituent of the atmosphere.

In their paper on "High-Frequency Absorption Phenomena in Liquids and Solids", Prof. Willis Jackson and Dr. J. A. Saxton directed attention to the significance of investigations on such materials as polythene, which is substantially non-polar, and liquid water, which exhibits purely polar characteristics. Such centimetre-wave measurements have served to illustrate more convincingly than previous work at longer wave-lengths the limitations of the original dipole theory of dielectric absorption formulated by Debye. With the reasonable prospects now in view of the extension of the range of these measurements to the millimetre wave-band and so towards the far infra-red portion of the spectrum, such investigations present interesting possibilities of leading to a fuller understanding of the properties of the liquid and solid states. In the meantime, the data already acquired on the absorption and scattering effects of water, ice and water vapour are of great value in connexion with the use of centimetre wave transmission through the atmosphere, and with the effect of fog, clouds, rain and snow on such transmission.

In the course of another paper in this session, Drs. R. Cooper and W. A. Prowse described experimental determinations of the minimum electric breakdown stress in air at frequencies corresponding to centimetre wave-lengths. It was found that radioactive irradiation of the spark gap improved the precision of the measurements without affecting the deductions drawn from the results. Spectrographic observations were made of the spark itself, and of the accompanying bright spot on the electrodes.

A paper entitled "Investigations of Magnetic Phenomena at Centimetre Wave-lengths" was presented by Dr. J. H. E. Griffiths, of the Clarendon Laboratory, Oxford, in which he stated that it is only recently that it has been possible to make measurements on the permeability of ferromagnetic materials in the centimetre wave region, and these have led to some interesting results. In addition to studying the permeability, which decreases rapidly with increase of frequency, the effect of a superimposed steady, transverse magnetic field has been investigated. It is then found that ferromagnetic metals exhibit a resonance phenomenon, in that the high-frequency loss in the metal becomes a maximum for a certain value of the steady magnetic field. A number of paramagnetic salts show a similar resonance absorption, and the interpretation of the results of such measurements is leading to a better understanding of the magnetic properties exhibited by various substances.

The last paper in the session, and also of the Convention, was presented by Mr. A. B. Pippard, 
who described experiments on the high-frequency conductivity of metals at low temperatures. It is well known that the classical theory, supported by measurements at moderately high frequencies, indicates that the resistivity of a conductor as determined by the skin effect should be inversely proportional to the square root of the bulk conductivity. Experiments made at a frequency of $1,200 \mathrm{Mc}$./s. have shown, however, that while this is true at room temperature, the great increase in conductivity due to cooling pure metals to very low temperatures is not accompanied by a corresponding decrease of surface resistivity. The explanation of this effect is that the free paths of the conduction electrons at low temperatures may exceed the classical value of the skin depth by as much as a factor of 100 under the conditions of the experiments. Thus it is no longer permissible to assume the validity of Ohm's law, since only a small proportion of the relevant electrons contribute to the surface con. ductivity. It is clear that we have here a quantitative radio technique for exploring the free paths of electrons, and investigations were referred to in which the method is being applied to the study of the surface characteristics of pure metals.

In the course of the discussion, Dr. R. A. Smith very aptly referred to the interesting fact that physicists, who had so ably assisted the radio investigators in the development and exploitation of radar and communications during the War, have now taken back into their own research laboratories some of the problems exposed by the radio engineers. The development of the higher frequency radio techniques in the past few years has clearly provided powerful research tools for studying the general physical constitution of matter.

R. L. SMITH-Rose

\section{PROBLEMS OF TIDAL BIOLOGY}

$I^{N}$ N a letter published in Nature in $1944(154,300)$ it was pointed out by Prof. T. A. Stephenson, of University College, Aberystwyth (in continuation of an argument initiated by Prof. F. E. Fritsch, Nature, $154,144 ; 1944)$, that future investigation of intertidal biology in Great Britain demanded : $(a)$ further work on the British coasts between tide-marks; (b) further work on the continental shelf of the British region below tide-marks ; and (c) an attempt to make a preliminary world statement, based on a carefully selected series of surveys made by the same workers.

Since 1944 progress has been made towards the realization of all these objectives, but the present article is concermed particularly with the third one. Prof. Stephenson has now undertaken to write a monograph on plant- and animal-life between tidemarks throughout the world, and the expedition described below was a step in that direction. The monograph is intended to supplement the well-known works of Ekman ("Tiergeographie des Meeres", 1935) and of Sverdrup, Johnson and Fleming ("The Oceans", 1942). These volumes both provide worldwide pictures of certain aspects of marine life, but neither devotes particular attention to the intertidal region; nor is there any general work which attempts to do this. In the task which he has under. taken, Prof. Stephenson will have behind him thirty years experience of ecological work between tidemarks carried out in Australia, South and East
Africa, Mauritius and Great Britain, much of which is embodied in a series of admirable papers. The shores of 'North America have now been added to the regions studied by him. In much of this work, of a type which could not be conducted unaided, Prof. Stephenson has been assisted by collaborators, and throughout Mrs. Stephenson has acted as his technical assistant.

Between December 1946 and January 1948, Prof. and Mrs. Stephenson made a thirteen months visit to the United States and Canada to collect new material for their monograph. It has been recognized throughout that, while nobody can expect to visit all the coasts of the world in this connexion, there is a certain amount of uniformity between one coast and another within the same temperature limits. There is thus reason to expect that a judiciously chosen series of samples will provide a basis for a general statement which, if only a first approximation, will form a foundation for later work. It so happens that most of the samples needed to complete Prof. and Mrs. Stephenson's earlier experience are to be found in North America. On this ground their visit was supported either financially or by the provision of facilities, or both, by a number of institutions in Great Britain and America.

While the intertidal ecology of the North American coasts is much better known than that of South America, it has been investigated less than might be supposed. There is an extensive literature dealing with the systematics of intertidal plants and animals, and a very considerable amount which describes the biology of particular species. Contributions reviewing the shore ecology as a whole are much fewer, and where they exist, may deal primarily with animals (for example, Hewatt ${ }^{1}$ ) or with plants (for example, Doty $^{2}$ ) and rarely cover wide areas, except in occasional works such as that of Ricketts and Calvin ${ }^{3}$, and the paper by Doty already mentioned. There are vast stretches of the coast which remain almost unknown from an ecological point of view. The places visited during the present work were chosen, so far as possible, in areas where the general shore ecology has not been described. The summary which follows is derived from a privately printed report prepared for submission to the institutions which supported the work.

The time spent in America extended from January 1, 1947, to January 25, 1948. During this period a distance of some 15,000 miles was covered, mainly by motor-car, and shore-work was carried out first on the Atlantic coast-among the Florida Keys, at Marineland in North Florida, at Charleston in South Carolina and at Beaufort in North Carolina. After crossing the continent it was continued on the Pacific coast at Nanaimo in British Columbia, and afterwards at Pacific Grove and La Jolla in California. This schedule permitted a stay of approximately two months at each of the main centres.

The amount of information collected was considerable, and it will be some time before it can be worked up for detailed publication. Meanwhile the following note will indicate the nature of the results. The work falls roughly under three headings: $(a)$ broad geographical aspects; $(b)$ questions of vertical and horizontal distribution within small areas; and $(c)$ the causes of the facts observed under $(a)$ and $(b)$.

The Florida Keys represent a tropical region with an abnormally scanty intertidal population; scanty, that is to say, in total bulk of organic material regarded as a crop, not in number of species repre. 